\title{
Septicemic Listeriosis in Wild Hares from Saskatchewan, Canada
}

\author{
Jamie L. Rothenburger, ${ }^{1,4}$ Katarina R. Bennett, ${ }^{2}$ Lorraine Bryan, ${ }^{3}$ and Trent K. Bollinger ${ }^{1,3}$ \\ ${ }^{1}$ Department of Veterinary Pathology, Western College of Veterinary Medicine, University of \\ Saskatchewan, 52 Campus Drive, Saskatoon, Saskatchewan S7N 5B4, Canada; ${ }^{2}$ Department of Small \\ Animal Clinical Sciences, Western College of Veterinary Medicine, University of Saskatchewan, 52 \\ Campus Drive, Saskatoon, Saskatchewan S7N 5B4, Canada; ${ }^{3}$ Canadian Wildlife Health Cooperative, \\ Western-Northern Region, University of Saskatchewan, 52 Campus Dr., Saskatoon, Saskatchewan S7N \\ 5B4, Canada; ${ }^{4}$ Current address: Department of Pathobiology, Ontario Veterinary College, University of \\ Guelph, 50 Stone Road East, Guelph, Ontario, Canada, N1G 2W1; ${ }^{5}$ Corresponding author (email: \\ jamie.rothenburger@gmail.com)
}

\begin{abstract}
The bacterium, Listeria monocytogenes, causes disease in a wide variety of mammals including rabbits and hares. We describe naturally acquired metritis and septicemic listeriosis in wild female hares from Saskatchewan, Canada. Between April 2012 and July 2013, two white-tailed jackrabbits (Lepus townsendii) and a snowshoe hare (Lepus americanus) were presented to the Veterinary Medical Centre at the Western College of Veterinary Medicine, Saskatoon, Saskatchewan, Canada with nonspecific neurologic signs. The hares were euthanized and autopsied. Necrotizing fibrinosuppurative metritis was present in all. Additional findings in individual hares included: fetal maceration, multifocal necrotizing myocarditis, multifocal hepatic necrosis, and nonsuppurative encephalitis. Listeria monocytogenes was cultured from multiple tissues in each hare. Although listeriosis in pregnant domestic rabbits has been studied, this is the first detailed description in wild North American hares. The epidemiology of listeriosis including prevalence and the role of environmental sources and coprophagy in transmission among hares requires further investigation.
\end{abstract}

Key words: Hare, Lepus americanus, Lepus townsendii, Listeria monocytogenes, listeriosis, metritis, septicemia.

The bacterium, Listeria monocytogenes, is remarkable in its ability to cause disease in mammals, birds and humans. Among domestic animals, $L$. monocytogenes most often infects ruminants, causing encephalitis, likely from contaminated silage (Roberts and Wiedmann 2003). Rabbits and hares (family Leporidae) are sporadically diagnosed with combined reproductive and septicemic listeriosis. Listeriosis was probably first described in two unidentified rabbits with multifocal hepatic necrosis in 1910 (Hülpers 1911). Listeriosis has been reported in domestic rabbits (Oryctolagus cuniculus) from western Canada (Macdonald et al. 1972; Ayroud et al. 1991). Additionally, listeriosis has been studied in laboratory rabbits (Gray et al. 1955). Using passive surveillance, the apparent prevalence of clinical listeriosis in European hares (Lepus europaeus) ranges from $0.6 \%$ to $2.4 \%$, although the true prevalence is likely underestimated by these studies (Wuthe and Schönberg 1999; Haerer et al. 2001). In North America, there is only one report of listeriosis in wild hares and rabbits, consisting of septicemic listeriosis in wild snowshoe hares (Lepus americanus) from Newfoundland, Canada (McKercher and Archibald 1959).

Between April 2012 and July 2013, three wild, mature, female hares were presented to the Western College of Veterinary Medicine Veterinary Medical 
Centre, Saskatoon, Saskatchewan, Canada, with nonspecific neurologic signs. All three were euthanized because of poor prognosis and submitted to the Canadian Wildlife Health Cooperative (CWHC) for autopsy. Representative tissue samples were fixed in $10 \%$ buffered formalin, embedded in paraffin, sectioned at $5 \mu \mathrm{m}$, and stained with hematoxylin and eosin. Three tissues from each hare were inoculated on blood and MacConkey agar then incubated at $35 \pm 2 \mathrm{C}$, in a $5 \% \mathrm{CO}_{2}$ incubator, for 24 $48 \mathrm{~h}$. Isolated colonies were subcultured on Listeriaselective agar and subjected to confirmatory biochemical tests (Songer and Post 2005). Immunohistochemical staining was conducted at Prairie Diagnostic Services, Saskatoon, using the immunoperoxidase technique of Loeb (2004) with minor modifications. Protease XIV was used for enzymatic treatment and 3,3'-diaminobenzidine as the chromogen. The University of Saskatchewan Animal Use Protocol 20090014 approved all procedures.

Hare 1, a snowshoe hare from Saskatoon $\left(52^{\circ} 7.8^{\prime} \mathrm{N}, 106^{\circ} 40^{\prime} \mathrm{W}\right)$ was found unable to move. The hare was in poor body condition and remained in left lateral recumbency during physical examination. The hare was moribund, nonresponsive to stimuli and bradycardic. At autopsy, the hare had no obvious fat stores. The uterus contained five macerated fetuses, which were surrounded by a thick yellow exudate (Fig 1). The uterine wall adjacent to the fetuses was hyperemic and multifocal areas on the serosal surface were covered by fibrinous exudate. In one area, the small intestine was firmly adhered to the uterine serosa.

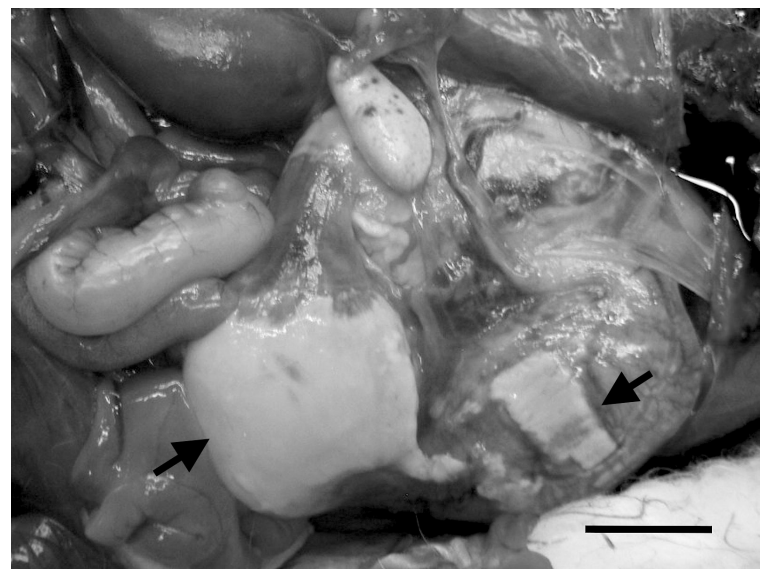

Figure 1. Listeria monocytogenes infection in the gravid uterus of a wild snowshoe hare (Lepus americanus) from Saskatoon, Saskatchewan, Canada (Hare 1). Thick yellow exudate coating the fetuses is visible through the uterine serosa (arrows). Bar $=2 \mathrm{~cm}$.
Microscopically, there was severe, necrotizing fibrinosuppurative metritis, with necrosis and fibrin exudation extending from the lumen through the endometrium and multifocally into the myometrium (Fig 2a). The endometrium and myometrium were multifocally mineralized. Many heterophils infiltrated the myometrium and extended to the fibrin-covered serosal surface. Within the brainstem, there was multifocal malacia with perivascular lymphocyte cuffs. Immunohistochemistry identified few, small, intracellular, short rods consistent with $L$. monocytogenes in these brainstem lesions (Fig 3). There was diffuse pulmonary edema and congestion. Listeria monocytogenes was isolated from kidney $(4+)$, spleen $(1+)$ and fetal lung $(2+)$.

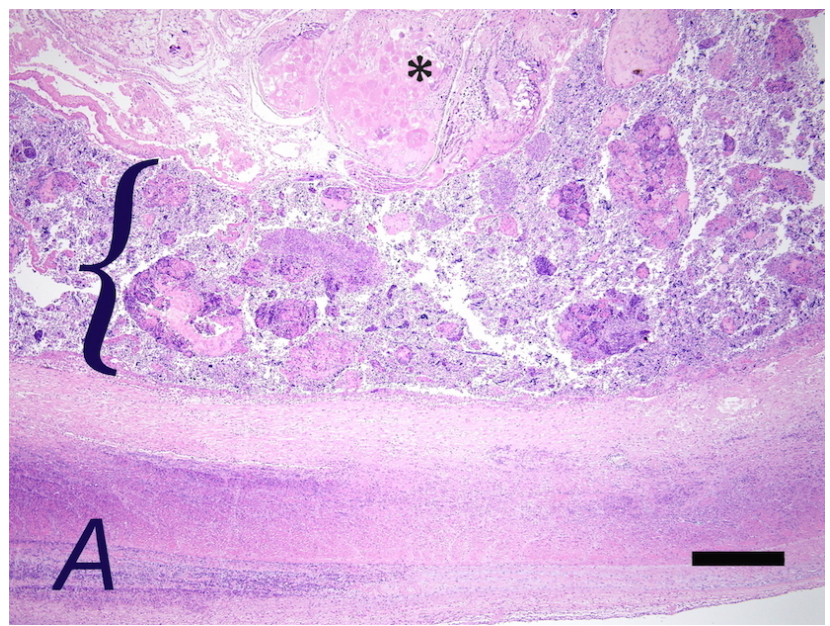

Figure 2a. Histologic features of Listeria monocytogenes infection in the gravid uterus of a wild snowshoe hare (Lepus americanus) from Saskatoon, Saskatchewan, Canada (Hare 1). Necrotic placenta (*) and cellular debris fills the lumen $(\{)$. H\&E stain. 4X; Bar=500 $\mu \mathrm{m}$.

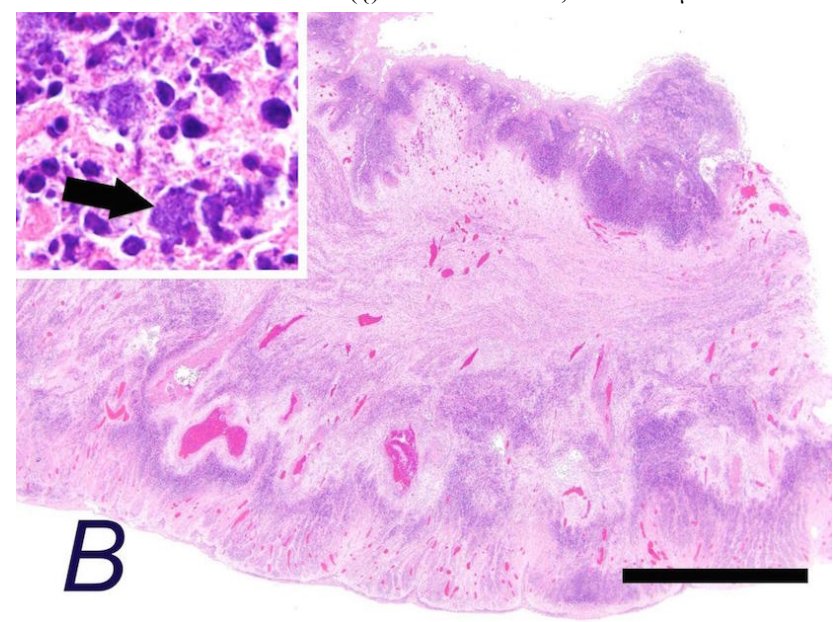

Figure 2b. Histologic features of transmural fibrinonecrotizing metritis in a white-tailed jackrabbit (Lepus townsendii) from Saskatoon, Saskatchewan, Canada (Hare 2) infected with Listeria monocytogenes. $\mathrm{H} \& \mathrm{E}$ stain. $2 \mathrm{X}$; Bar=2 mm. Inset: necrotic cell debris with multiple bacterial colonies (arrow). 


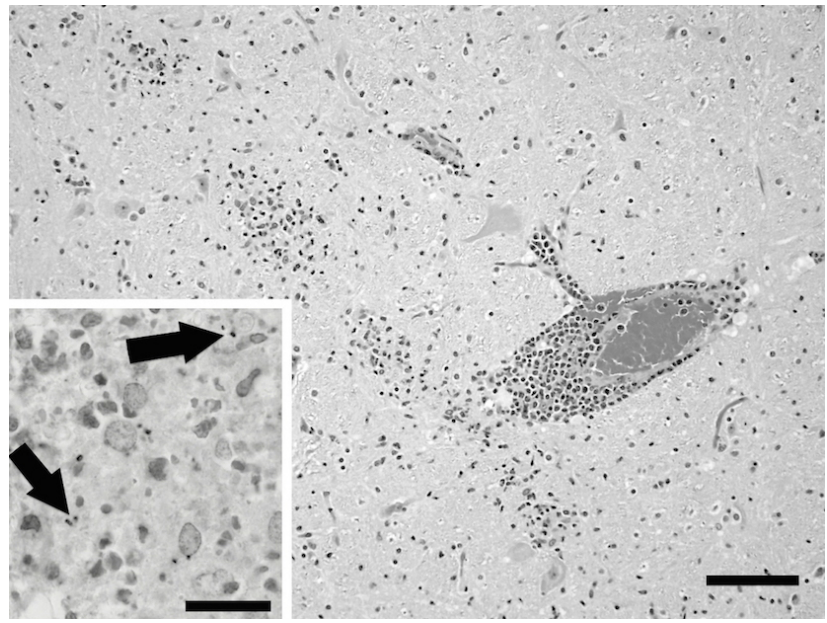

Figure 3. Histologic features of encephalitis associated with Listeria monocytogenes in the brain of a wild snowshoe hare (Lepus americanus) from Saskatoon, Saskatchewan, Canada (Hare 1). There is multifocal malacia, gliosis, and lymphocytic perivascular cuffing. H\&E stain. 20X; Bar=100 um. Inset: immunohistochemistry highlights individual $L$. monocytogenes bacteria in a focus of malacia (arrows). Avidin-Biotin complex immunoperoxidase technique with hematoxylin counter stain. 100X; Bar $=20 \mu \mathrm{m}$.

Hare 2, a white-tailed jackrabbit (Lepus townsendii) from an urban park in Saskatoon, was presented with neurologic signs including continual rolling of the body to one side and horizontal nystagmus of both eyes. The hare was nonresponsive to stimuli. At autopsy, the hare was in good body condition. Within the uterus, there was multifocal congestion, hemorrhage and fibrin attached to the endometrial surface. There were multiple fibrin tags throughout the peritoneal cavity. The urinary bladder was severely distended and contained approximately $300 \mathrm{~mL}$ of urine with flocculent, yellow material. Mammary glands and nipples were prominent, indicating lactation. Throughout the myocardium, there were multifocal, 1-10 $\mathrm{mm}$ pale areas (Fig 4).

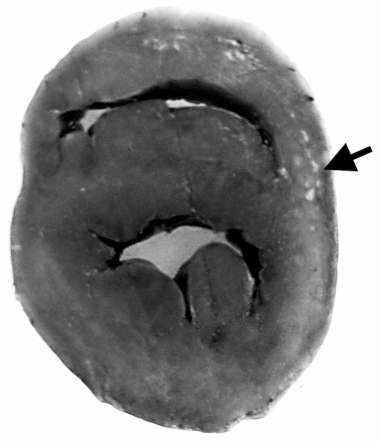

Figure 4. Heart from a white-tailed jackrabbit (Lepus townsendii) from Saskatoon, Saskatchewan, Canada (Hare 2). Multifocal pale areas (necrotizing myocarditis) are present throughout the myocardium (arrow).
Histopathology of the uterus was similar to hare 1 (Fig 2b). Additionally, there was multifocal vascular thrombosis in the myometrium. There was multifocal myocardial necrosis and myocarditits comprised of heterophils, lymphocytes, plasma cells, and macrophages (Fig. 5a). Random, multifocal areas of necrosis were present in the liver (Fig. 5b). Bacterial colonies were evident within lesions in the heart and liver and were also present in the renal tubules, spleen, adrenal gland, diaphragm, small intestine, and lymph nodes. Listeria monocytogenes was cultured from the uterus $(2+)$, heart $(2+)$ and spleen $(4+)$.

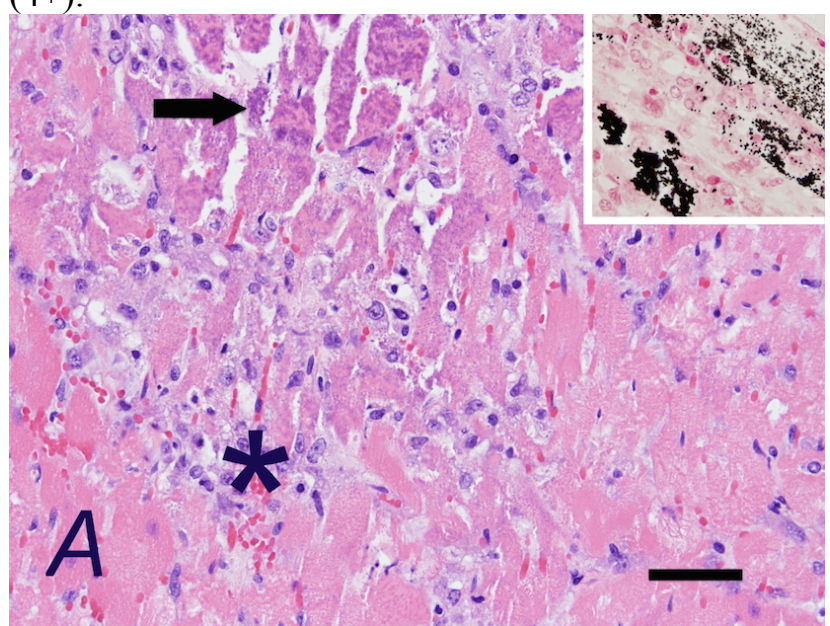

Figure 5a. Histologic features of necrotizing myocarditis in a whitetailed jackrabbit (Lepus townsendii) from Saskatoon, Saskatchewan, Canada (Hare 2) infected with Listeria monocytogenes. Affected areas are infiltrated by macrophages and lymphocytes $(*)$. Multifocally, there is cardiac myocyte fragmentation and mineralization (arrow). H\&E stain. 40X; Bar $=50 \mu \mathrm{m}$. Inset: mineralized cardiac myocytes (black pigment).

Von Kossa stain.

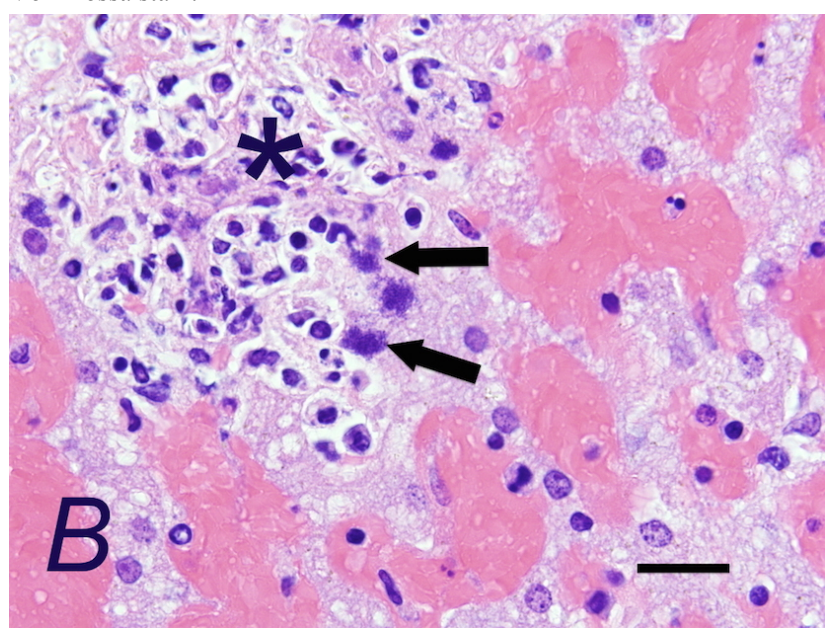

Figure $5 \mathrm{~b}$. Histologic features of necrotizing hepatitis in a white-tailed jackrabbit (Lepus townsendii) from Saskatoon, Saskatchewan Canada (Hare 2) infected with Listeria monocytogenes. Bacterial colonies (arrows) are adjacent to necrotic hepatocytes $(*)$. H\&E stain. 100X; Bar $=20 \mu \mathrm{m}$. 
Hare 3, a white-tailed jackrabbit from Warman, Saskatchewan $\left(52^{\circ} 19^{\prime} \mathrm{N}, 106^{\circ} 34^{\prime} \mathrm{W}\right)$ was found unable to ambulate. Clinical signs included bradycardia, obtundation and both hindlimbs were held forward in rigid extension. Nipples were prominent suggestive of lactation. Pain was elicited when the lumbar spine area was palpated and the urinary bladder was distended and difficult to express. The urine was dark orange-red, and urine strip analysis revealed 4+ hemoglobin. A spinal cord injury was suspected and the hare was euthanized. Hare 3 was lactating and in poor body condition with no visible fat stores. The uterus was flaccid, dilated, and contained necrotic debris within the lumen. The serosal surface was dark red. There was a focal 1-2 $\mathrm{cm}$ area of necrosis in the left semimembranosis and semitendinosis muscles, increased laxity in the left sacroiliac joint and hemorrhage in the vertebral canal at the thoracolumbar junction and sacrum that was attributed to trauma. The urinary bladder was distended and contained dark brown urine. The lungs were diffusely edematous with froth in the trachea and bronchi. Histopathology of the uterus was similar to hare 1 , with the addition of multifocal vascular thrombosis in the myometrium. There was diffuse pulmonary edema and congestion. Listeria monocytogenes was cultured from the uterus $(2+)$, lung (few), and spleen (1+). The uterus and lung also had low growth of Escherichia coli (1+).

We searched the CWHC database for similar cases in wild hares and rabbits. No other cases of listeriosis were identified among 340 Leporidae submissions.

Gross and microscopic lesions, in conjunction with bacterial culture, indicate the three hares were suffering from septicemic listeriosis secondary to metritis. This is in agreement with previous studies of listeriosis in domestic rabbits and European hares, which describe similar combinations of metritis, with or without macerated fetuses, multifocal hepatic necrosis, and peritonitis (Gray et al. 1955; Wuthe and Schönberg 1999). Multifocal hepatic necrosis is a nonspecific finding since it is also seen in other causes of septicemia in rabbits and hares, including: Clostridium piliforme, Francisella tularensis, Pasteurella multocida and Salmonella spp. (Percy and Barthold 2007).

Unusual lesions in these hares include myocarditis and encephalitis. Myocarditis occurs sporadically in other species with listeriosis (Dustoor et al. 1977; Lecuit 2007; Crespo et al. 2013), but has not been described in rabbits or hares. Encephalitis has not been previously described in North American hares with listeriosis. Encephalitis is rare in rabbits and European hares with listeriosis but is the typical manifestation in ruminants (Wuthe and Schönberg 1999; Percy and Barthold 2007).

Pregnant domestic rabbits are uniquely susceptible to listeriosis. After oral inoculation, pregnant does aborted or developed metritis or septicemia while nonpregnant does and males did not (Gray et al. 1955). In rabbits, the pathogenesis of infection includes intestinal invasion, hematogenous spread and breach of the placental barrier, leading to metritis, placentitis, fetal maceration, and ultimately abortion (Gray et al. 1955). Listeria monocytogenes can be isolated from placenta and fetal tissues as early as $30 \mathrm{~h}$ after oral exposure (Gray et al. 1955). A similar pathogenesis is suspected in these hares and is supported by a previous study, which found that $78 \%(7 / 9)$ of wild European hares with listeriosis were peripartum does (Wuthe and Schönberg 1999). In addition to pregnancy, other immunosuppressive host factors, such as stress, predispose animals and humans to listeriosis (Roberts and Wiedmann 2003).

Although the source of infection in these cases is unknown, oral transmission from environmental sources including food is probable. As an environmental contaminant, $L$. monocytogenes is found worldwide and can survive in adverse conditions such as low temperatures, extreme $\mathrm{pH}$ levels, and high salinity (Roberts and Wiedmann 2003).

The role of coprophagy in L. monocytogenes transmission among leporids has never been investigated. Autocoprophagy, routine consumption of the individual's own hard and soft feces, is an important nutritional adaptation, allowing exploitation of poorly-digestible food and provision of vital nutrients and energy during times of starvation (Hirakawa 2001). In laboratory mice, allocoprophagy is not an apparent mode of transmission (Conlan 1997). Given that $L$. monocytogenes is shed in feces of healthy animals (Roberts and Wiedmann 2003), and that oral transmission in domestic rabbits produces listeriosis (Gray et al. 1955), it is possible that carrier animals may become systemically infected through autocoprophagy during the window of susceptibility 
provided by pregnancy. This mode of transmission warrants further investigation.

The zoonotic risk from $L$. monocytogenes in hares is unknown. Although listeriosis is among the most common of food-borne illnesses, infection is typically associated with contaminated fruit and deli meat, with only sporadic cases reported from contact with infected animals (Roberts and Wiedmann 2003).

It is unlikely that this cluster of listeriosis represents disease emergence, but rather a sporadic cause of mortality in hares. These hares were found sick in urban centers. Rather than a true risk factor, this likely reflects the increased chance of people finding sick or dead wildlife in cities compared to rural or natural environments. All three hares were debilitated, which would increase susceptibility to trauma, as was seen in one hare.

In Switzerland, vehicle-associated trauma was a leading cause of mortality, with only $15 \%(25 / 167)$ of cases attributed to infectious disease, including listeriosis (Haerer et al. 2001). The true prevalence of listeriosis in wild hares in North America is unknown but is likely low. Further research will be necessary to understand species susceptibility, the impact of listeriosis on populations, and modes of transmission.

We thank Christine Wilson for assistance with the Canadian Wildlife Health Cooperative Database search, LaRhonda Sobchishin for help with image preparation, Dale Godson for immunohistochemistry, and Gary Wobeser for helpful comments during preparation of this manuscript.

\section{LITERATURE CITED}

Ayroud M, Chirino-Trejo M, Kumor ML. 1991. Saskatchewan. Listeriosis in rabbits. Can Vet J 32:44.

Conlan JW. 1997. Neutrophils and tumour necrosis factor-alpha are important for controlling early gastrointestinal stages of experimental murine listeriosis. J Med Microbiol 46:239-250.

Crespo R, Garner MM, Hopkins SG, Shah DH. 2013. Outbreak of Listeria monocytogenes in an urban poultry flock. BMC Vet Res 9:204.

Dustoor M, Croft W, Fulton A, Blazkovec A. 1977. Bacteriological and histopathological evaluation of guinea pigs after infection with Listeria monocytogenes. Infect Immun 15:916-924.
Gray ML, Singh C, Thorp F. 1955. Abortion, stillbirth, early death of young in rabbits by Listeria monocytogenes. II. Oral exposure. Proc Soc Exp Biol Med 89:169-175.

Haerer G, Nicolet J, Bacciarini L, Gottstein B, and Giacometti M. 2001. Todesursachen, Zoonosen und Reproduktion bei Feldhasen in der Schweiz. [Causes of death, zoonoses, and reproduction in the European brown hare in Switzerland]. Schweiz Arch Tierheilkd 143:193-201.

Hirakawa H. 2001. Coprophagy in leporids and other mammalian herbivores. Mamm Rev 31:61-80.

Hülpers G. 1911. Liver necrosis in rabbit caused by a hitherto unknown bacterium. Svensk Veterinartidskrift 15:2:265-273 (in Swedish). In: International symposium on problems of listeriosis $X V$. Uppsala, Sweden, 12-15 September 2004, abstract 147a (in English).

Lecuit M. 2007. Human listeriosis and animal models. Microbes Infect 9:1216-1225.

Loeb E. 2004. Encephalitic listeriosis in ruminants: Immunohistochemistry as a diagnostic tool. $J$ Vet Med A Physiol Pathol Clin Med 51:453-455.

Macdonald DW, Wilton GS, Howell J, Klavano GG. 1972. Listeria monocytogenes isolations in Alberta 1951-1970. Can Vet J 13:69-71.

McKercher PD, Archibald RM. 1959. Listeriosis in the Atlantic provinces. Can J Comp Med Vet Sci 23:274-275.

Percy DH, Barthold SW. 2007. Rabbit. In Pathology of laboratory rodents and rabbits, $3 \mathrm{rd} \mathrm{Ed}$.

Blackwell Publishing, Ames, Iowa, pp. 253-307.

Songer JG, Post KW. 2005. The genera Listeria and Erysipelothrix. In: Veterinary Microbiology. Elsevier Saunders, St. Louis, Missouri, pp. 8794

Roberts AJ, Wiedmann M. 2003. Pathogen, host and environmental factors contributing to the pathogenesis of listeriosis. Cell Mol Life Sci 60:904-918.

Wuthe, HH, and Schönberg A. 1999. Listeriose beim Feldhasen in Norddeutschland. [Listeriosis in the European brown hare in northern Germany]. Berl Münch Tierärztl Wochenschr 112:98-99. 